\title{
Trabajo infantil doméstico y violencias en la ciudad de Tapachula, Chiapas 2017-2018
}

\section{Domestic child labor and violence in the city of Tapachula, Chiapas 2017-2018}

\author{
Sarai Miranda Juárez \\ El Colegio de la Frontera Sur, Unidad San Cristóbal de las Casas, México
}

\section{Resumen}

El objetivo del artículo es identificar las violencias que se intersectan contra niñas y adolescentes trabajadoras domésticas en la ciudad de Tapachula, Chiapas. Se enfatiza en las violencias raciales asociadas al origen étnico y la condición migratoria. La metodología aplicada es cualitativa e incluyó diversas estrategias puestas en marcha de julio de 2017 a julio de 2018. Se aplicaron entrevistas semi-estructuradas a niñas y adolescentes inmigrantes e indígenas procedentes de Guatemala que realizan trabajo doméstico en hogares de terceros y a otros actores involucrados. Se observaron reiteradas violencias a razón de la etnia, género, edad y condición migratoria, que se ejercen en un contexto local caracterizado por inercias globales imbuidas de miedo e inseguridad frente a lo extranjero. Se muestra la renovación de discursos xenofóbicos y estigmatizadores por parte de las oligarquías y clases medias locales, que al ejercer violencias se benefician de trabajo doméstico y de cuidado a muy bajo costo.

Palabras clave: Violencias, trabajo infantil doméstico, Tapachula, México.

\section{Abstact}

This article's objective is to identify violences that intersect among domestic workers girls and adolescents in the city of Tapachula, Chiapas. It makes a special emphasis on the racial violence associated with the ethnical origin and migratory condition. The study applied a quantitative methodology, including several strategies implemented from July 2017 to July 2018 . Semi-structured interviews were applied to indigenous and inmigrant girls and adolescents from Guatemala who perform domestic work in third-party households as well as to other involved actors. The research observed systematic violences related to ethnicity, gender, age, and migratory condition. Such violences are exercised in a local context characterized by global inertia permeates by fear and insecurity concerning the foreign. The article also shows the renewal of xenophobic and stigmatizing discourses of the oligarchy and the middle classes, which at exerting violence benefit themselves of domestic work and care at a very low cost.

Key words: Violences, domestic workers girls, Tapachula, Mexico.

${ }^{1}$ Este artículo forma parte de una serie de publicaciones derivadas de un trabajo de investigación titulado "Migración, trabajo doméstico y violencia de género. Niñas, niños y adolescentes en el sureste de México" que contó con el financiamiento de la Beca a las Mujeres en las Ciencias Sociales de la Academia Mexicana de Ciencias 2017-2018. 


\section{INTRODUCCIÓN}

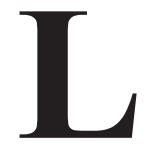

a región fronteriza entre México y Guatemala ha sido considerada como "una frontera sui géneris en tanto que no existen muros, vallas o alambrados que separen e impidan el paso de población centroamericana hacia México" (Nájera, 2016: 256). La relación histórica que guardan ambos países ha generado una zona de alta movilidad poblacional caracterizada por la existencia de cruces fronterizos heterogéneos que responden a diversas necesidades tanto económicas como sociales y políticas. Recientemente es también considerada una de las fronteras más violentas y mortales del mundo (París, 2017).

Si bien, la configuración de la frontera tiene una historia que data desde el siglo XIX cuando se establecieron los límites internacionales, los procesos de movilidad humana habían estado signados por comunidades que compartían relaciones interétnicas y una vida cotidiana con relativa libertad de circulación (Cortez, Cáceres y Venegas, 2005). Por diversos factores de orden económico y político-administrativos, la frontera (en particular el territorio que corresponde al estado de Chiapas) se volvió sumamente dinámica y se configuró como una de las fronteras más importantes en la actualidad dada la complejidad de sus patrones migratorios. En este sentido, la frontera de Chiapas se llegó a denominar como una frontera altamente porosa pues para finales de 2009 se estimaba que ingresaban un millón de migrantes anualmente a través de los ocho cruces formales (puentes internacionales) y 4,313 pasos informales, además de los que se atravesaban a pie por caminos de extravío (Ramírez, 2009).

Hoy en día, el panorama es mucho más complejo. Diversas razones impulsan la movilidad humana en esta frontera: a la migración tradicional de indígenas guatemaltecos que se siguen movilizando en busca de trabajo en las fincas agrícolas en la región del Soconusco; se le ha sumado la migración de tránsito de población centroamericana que busca llegar a Estados Unidos como destino final, y últimamente es la puerta de entrada también de migrantes procedentes de África, Haití, Cuba y otras regiones del mundo que desean huir de condiciones de marginalidad o de violencia en sus territorios de origen (OIM, 2018).

Este panorama ha derivado en nuevas políticas migratorias por parte del Estado mexicano con el objetivo de controlar sus fronteras. Un ejemplo de ello es la puesta en marcha del Programa Frontera Sur en el año 2014, con lo que se incrementó la inversión en infraestructura que limita el tránsito de personas. El resultado ha sido un incremento abrumador de las detenciones 
de 2013 a 2015, que pasó de más de 86 mil a más de 198 mil (OIM, 2018). Las propias autoridades mexicanas han reconocido que esta frontera presenta altos índices de inseguridad para los inmigrantes centroamericanos, entre otras razones por la presencia cada vez más extendida de redes de narcotráfico (Porraz, 2017).

En este contexto de suma complejidad que se ha tornado mucho más hostil, siguen coexistiendo flujos migratorios tradicionales con las nuevas formas de movilidad. Las niñas, los niños y las y los adolescentes son actores sociales que han estado presentes en esta frontera y día a día se movilizan en busca de trabajo y mejores condiciones de vida, ya sea solos o con su grupo familiar. Entre ellos la niñez indígena guatemalteca ha encontrado en la ciudad de Tapachula diversos nichos de mercado donde se emplean y obtienen recursos económicos para su sustento cotidiano. La venta de dulces, así como la limpieza de zapatos en los principales parques y mercados de la ciudad son actividades que se nutre de niños y adolescentes varones, mientras que el trabajo doméstico en hogares de terceros es la actividad donde más participan las niñas y las adolescentes guatemaltecas (López, 2018, Blanco, 2014).

El trabajo doméstico que realizan las niñas y las adolescentes indígenas e inmigrantes guatemaltecas en territorio mexicano, se inserta en las tendencias actuales que permiten que se internacionalicen las tareas domésticas y de cuidados y que sean asignadas a trabajadoras inmigrantes, caracterizadas por portar marcadores sociales como el género, la etnia, la condición migratoria y la edad (Anderson, 2000). Las cadenas globales de cuidados se sustentan en la interrelación tanto de la división sexual del trabajo como del origen migratorio de las mujeres, las jóvenes y las niñas que realizan dichas tareas, "la población nativa ya no se encuentra dispuesta a realizar este tipo de actividad en función de las desfavorables condiciones laborales y económicas que presenta, así como de su escaso prestigio social con reminiscencias de situaciones de servilismo y miseria" (Díaz, 2008: 75). Además, el modelo de servidumbre basado en el binomio superioridad-inferioridad entre grupos raciales específicos parece haber renovado su vigencia con la globalización y la migración (Hondagneu, 2011).

Pese a que no existen diagnósticos de gran extensión territorial y temporal, se sabe que quienes realizan trabajo doméstico y de cuidados para terceros se enfrentan a las peores condiciones laborales, sufriendo condiciones de explotación, abuso, maltrato, discriminación, falta de pago, largas jornadas, limitadas oportunidades educativas y aislamiento de las familias (Ramellini, 2004). De igual forma, las niñas y las adolescentes 
comúnmente nombradas como niñas de casa, niñas de crianza, sirvientas, criadas; están frecuentemente expuestas a la violencia sexual y de género (OIT-IPEC, 2002).

El presente artículo tiene por objetivo describir y analizar las diferentes violencias que experimentan las niñas y las adolescentes que realizan trabajo infantil doméstico en hogares de terceros en la ciudad de Tapachula, Chiapas. Se pone especial énfasis en las violencias asociadas a procesos de racialización.

Para el logro del objetivo, se emplea una metodología de corte cualitativo, a fin de identificar el sentido que dan los individuos a la práctica del trabajo infantil doméstico, se busca echar mano de las subjetividades individuales frente a las violencias ejercidas y sufridas, realidades entendidas como formas de subjetividad socialmente construidas que expresan especificidades de cada actor social, la forma en que experimenta su realidad y la forma en que despliega diversas estrategias y prácticas para sobrevivir de la mejor manera posible (Glaser y Strauss, 1967).

La estrategia metodológica se conformó por diferentes herramientas, tales como observación participante en el Parque Central de Tapachula y en los mercados, entrevistas semiestructuradas con niñas y adolescentes inmigrantes que se emplean en el servicio doméstico, empleadoras, autoridades consulares, autoridades de los centros de salud, entre otros actores involucrados. El trabajo de campo se llevó a cabo durante tres estancias en la ciudad entre diciembre de 2017 y mayo de 2018.

\section{SOBRE NIÑAS Y ADOLESCENTES INMIGRANTES E INDÍGENAS QUE REALIZAN TRABAJO DOMÉSTICO}

En América Latina, el estudio metódico sobre la movilidad de la población ha estado particularmente presente desde la segunda mitad del siglo XX, justo cuando los especialistas dieron cuenta de gran parte de las dimensiones sociales y económicas involucradas en la movilidad poblacional. En términos generales, la literatura teórica y empírica comenzó a rastrear los vínculos entre la migración y las características sociodemográficas, económicas y productivas en las áreas de origen y de destino (Solimano, 2001; IMF, 1994). Por otra parte, se buscó la relación entre las lógicas individuales y mesosociales, sobre todo de las unidades domésticas, que tomaban las decisiones migratorias y valoraban los costos y beneficios de la movilidad de la fuerza de trabajo familiar en el mercado local, regional o internacional (Bustamante, 2008). La migración también fue estudiada a partir de la 
centralidad de la construcción de redes sociales entre las personas migrantes (Borjas, 1999; Hatton y Williamson, 1994).

A partir de las décadas conocidas como de corte neoliberal, la literatura sobre la migración dio cuenta de nuevos actores involucrados. Un ejemplo fue la notoria visibilidad que se dio a la migración femenina, con lo cual se incorporó la perspectiva de género en la forma de analizar la movilidad de la población. La presencia de niños, niñas y adolescentes en los estudios sobre migración tanto regional como internacional, se ha dado más recientemente, sobre todo bajo la novedosa hipótesis de que la migración de estos nuevos actores no está necesariamente ligada a las unidades domésticas (Ramírez, 2009;). La presencia de niños, niñas y adolescentes migrantes no acompañados es uno de los retos más profundos que ha enfrentado el campo de estudio de las migraciones. Este nuevo tópico se cruza con otras dimensiones que complejizan el análisis. Las indagaciones sobre la niñez migrante se vinculan directamente con la vulneración de los derechos más fundamentales cuando se trata de niñez refugiada, niñez desplazada, o bien cuando se trata de movilidad con fines de explotación laboral y sexual (Casillas, 2006). Se ha documentado también la existencia de adolescentes que deciden desplazarse sin que medie algún tipo de coerción (Ramírez, 2009). Dentro del cúmulo de recientes investigaciones sobre niñez y adolescencia migrante, se encuentran quienes han puesto el foco de atención en el vínculo entre migración y trabajo, partiendo del hecho de que mundialmente siguen siendo significativa la cantidad de adolescente que trabajan. Las investigaciones analizan casos de migración rural-rural y rural-urbana de niños, niñas y adolescentes, y discuten los motivos de la movilidad, las distintas formas de circulación, y los tipos de trabajo en que se emplean los niños y las niñas según sexo; la temporalidad y estacionalidad y las modalidades de pago (López, 2018; Miranda, 2016; Martínez, 2014; Glockner, 2008). El consenso general es que se trata de un tema inagotable que se complejiza al ritmo que se complejiza la realidad social, en tanto que se van reconociendo nuevos ejes de análisis en el marco de relaciones intergeneracionales, interétnicas, de clase y de género (Tijoux, 2013).

El aspecto laboral, especialmente el trabajo doméstico, sigue siendo un nicho donde se insertan niñas, niños y adolescentes. Las estimaciones de la OIT, señalan que entre 1990 y 2010 aumentó en 19 millones el número de empleados domésticos alrededor del mundo. Teniendo importante presencia "emigrantes de género femenino que iban buscando trabajo a países de Asia, de América Latina y de los países del Caribe" (Benítez, 2013: 2). El 90 por ciento de quienes realizan servicios domésticos son mujeres y niñas. 
Se estima que para 2008 en el mundo existían "7.4 millones de niñas, de niños y de adolescentes realizando servicios domésticos" (Benítez, 2013: 2).

La vulnerabilidad a la que se enfrentan las y los trabajadoras/es domésticos se profundiza cuando son niñas, niños y adolescentes. Se sabe que en algunos países africanos existe una modalidad de trabajo doméstico conocida como petites bonnes, que son aquellas niñas, niños y adolescentes que realizan labores domésticas en un hogar de terceros a cambio de la manutención cotidiana como un techo donde alojarse, comida y vestido; son mayoritariamente niñas migrantes que "trabajan con la puerta cerrada, en la intimidad de un hogar donde las posibilidades de explotación y abuso apenas son visibles" (Llorent, 2013: 355). De forma similar sucede en algunos países de Sudamérica con el sistema de criadrazgos, donde niños y niñas que migran de comunidades rurales hacia las ciudades entran en dinámicas "que implican la convivencia de una niña o un niño con una familia que no es la suya y que, a cambio de trabajo doméstico, le ofrece abrigo, educación y alimentación" (Muñoz y Ochoa, 2010: 70).

Esta práctica social ha sido estudiada desde diversas perspectivas. En primer lugar, se han realizado estudios y diagnósticos descriptivos sobre el trabajo infantil doméstico desde el punto de vista de los derechos de las niñas, niños y adolescentes (Alvarenga, Benítez y Walder, 2005), que pone en el centro del análisis la vulnerabilidad que se genera frente al ejercicio cotidiano de los derechos humanos y considera que "cuando una niña realiza trabajo doméstico en casa ajena se multiplican las posibilidades y maneras de violentar sus derechos como persona y como niña, quedando atrapada en una compleja red de poderosas fuerzas que la excluyen como actora y protagonista de su vida y su destino" (Carcedo, 2004: 29). Ejemplos de estos estudios son los diagnósticos realizados en Haití con los denominados restavekz y los petite bonnes en el norte de África (KidsRights Foundation, 2014; Association Al Kara, 2009; Human Right Watch, 2005).

Por otra parte, se encuentran los análisis que ponen en el centro de atención la perspectiva de género para visibilizar las desigualdades en que se encuentran las niñas y las adolescentes que realizan trabajo doméstico en hogares de terceros. Este enfoque parte de la necesidad de "desnaturalizar los roles tradicionalmente desempeñados por mujeres y por hombres" (Alvarenga, Benítez y Walder, 2005: 10). Igualmente, reconoce que las inequidades de género inician desde antes del nacimiento y se profundizan y adquieren sus dimensiones concretas durante la infancia y la adolescencia (Sagot, 2004). 
Desde esta óptica, el trabajo infantil doméstico es una extensión de la forma en que se asumen las tareas de cuidado en la sociedad, asignadas unilateralmente a las mujeres y dadas por extensión a las niñas y las adolescentes. Igualmente señalan que hay una sistemática invisibilización de dichas actividades con lo que se oculta el aporte de las niñas y las mujeres a la reproducción social y económica (Carcedo, 2004).

Un aporte sustancial desde este enfoque analítico es el reconocimiento de la vulnerabilidad de las niñas y las adolescentes frente a todo tipo de violencias, desde la psicológica hasta la sexual. Algunos estudios en el sur de América Latina han puesto de manifiesto que las niñas y las adolescentes que prestan servicios domésticos en casa ajenas a cambio de manutención se enfrentan a continuos atropellos a su sexualidad (Alvarenga, Benítez y Walder, 2005: 18): "estas niñas y adolescentes enfrentan el trato despectivo y discriminatorio, los abusos y frecuentes casos de explotación sexual, van horadando sus frágiles vidas y su autoestima" (Alvarenga, Benítez y Walder, 2005: 18).

Una tendencia mucho más reciente, que ha puesto en el centro del análisis a las mujeres y jóvenes es el enfoque de la Cadena Global de Cuidados. Esta perspectiva ha dado cuenta de la importancia de las nuevas formas de participación laboral de las mujeres y las niñas migrantes a partir de las migraciones femeninas de los países periféricos hacia los países centrales. Se reconoce que hay una división sexual y étnica del trabajo doméstico y de cuidados, por lo que son las mujeres y las jóvenes migrantes quienes desempeñan esas labores (Durin, 2017; Anderson, 2000).

$\mathrm{Al}$ respecto se encuentran las investigaciones realizadas en España con las mujeres y jóvenes migrantes que provienen de Sudamérica, principalmente de Perú. Estas investigaciones muestran la interrelación entre género y condición migratoria, afirmando que tanto la división sexual del trabajo como el origen migratorio de las mujeres y las jóvenes que realizan estas tareas son centrales para comprender los nuevos comportamientos en las cadenas globales de cuidados, con estudios de caso de mujeres jóvenes que migran de Perú hacia Chile y de Bolivia y Paraguay hacia Argentina (Arriagada y Moreno, 2011; Rodríguez, 2007).

Igualmente, los trabajos pioneros de Parreñas (2005) sobre inmigraciones hacia Europa de mujeres jóvenes que provienen de Filipinas empezaron a evidenciar el cruce entre género, clase y generación en la nueva organización global de la reproducción social, e incluye a las niñas y las adolescentes migrantes como actores sociales elementales en la cadena global de cuidados. 
De forma paralela, algunos desarrollos teóricos han puesto en el centro de la discusión y el análisis la forma en que se intersectan las categorías de edad, género y etnia en el contexto de las migraciones globales. Investigaciones basadas en niñez migrante dan cuenta de cómo los procesos de racialización no se agotan con el origen étnico de las personas, existe un correlato entre población migrante y racismo que tiene su fundamento en "la desnaturalización del otro mediante la figura del extranjero, del que se encuentra fuera de los límites de la comunidad, y del pobre, inferior económica y culturalmente; dando forma a un sujeto ajeno al proceso civilizatorio, elemento fundamental para comprender los procesos de construcción de lo nacional" (Tijoux y Palominos, 2015: 2).

En este sentido, los procesos migratorios tienen implicaciones relacionadas con la reproducción de jerarquías y estereotipos frente a ciertos grupos de población que migran. Estas jerarquías se fundamentan en la idea de la conformación de identidades nacionales donde los "otros" son considerados como inferiores al "nosotros" con lo que se justifican todo tipo de violencias (Tijoux y Palominos, 2015; Barot y Bird, 2001).

Algunos colectivos de inmigrantes son imaginados como marginales y subordinados, asociados con características físicas y costumbres sociales exóticas y difíciles de insertar a los ideales nacionales. Así, la extranjeridad se corporalizan en la figura del inmigrante y lo supedita a diversas exclusiones y dominaciones (Tijoux y Palominos, 2015; Barot y Bird, 2001). Tales jerarquías generan estereotipos como la supuesta "sensualidad y disponibilidad sexual de las mujeres racializadas, el temperamento arrogante de "negras" y "mulatas", o la sumisión y servilismo de las "indias" latinoamericanas" (Tijoux y Palominos, 2015: 6).

Al respecto, Hondagneu (2011) afirma que la cuestión racial y la migratoria se conjuntan y resultan en combinaciones novedosas donde la racialización deriva en una profundización de la racialización de las ocupaciones, "la inmigración no triunfa sobre la raza, pero al combinarse con la ideología dominante de una sociedad "ciega al color" logra, en cambio, encubrirla" (2011: 41).

Poco se sabe de lo que ocurre en México en relación al trabajo doméstico realizado por niñas y niños en hogares de terceros, mucho menos si son migrantes internacionales. Entre las escasas investigaciones se ha documentado la existencia de casos en los que las adolescentes procedentes de Guatemala hacen uso de su agencia y toman la decisión de migrar y trabajar como una opción ante la falta de mejores condiciones de vida en sus comunidades de origen (Blanco, 2014). 
El Módulo de Trabajo Infantil 2017 de la Encuesta Nacional de Ocupación y Empleo reporta que en México existen 20’992,495 personas de 5 a 17 años que realizan quehaceres domésticos (en sus propios hogares o en hogares de terceros), de los cuales 1'376,966 (siete por ciento), realizan dichas actividades en condiciones no adecuadas, con una marcada brecha de género en materia educativa ya que en inasistencia escolar 67.5 por ciento son mujeres y 32.5 por ciento hombres (INEGI, 2017).

La misma encuesta muestra que cinco por ciento de las personas de 5 a 17 años declararon ser "trabajadores domésticos, de limpieza, planchadores y otros" y 7.5 por ciento "otros trabajadores en actividades elementales"'; en entidades como Chiapas, Puebla y Michoacán con importante presencia de población indígena el mayor porcentaje es por género y se concentra en las de niñas y las adolescentes (INEGI, 2017). Lamentablemente son escasas las investigaciones que den cuenta e identifiquen las violencias asociadas a la condición migratoria y laboral de estas niñas y adolescentes en la frontera sur de México.

\section{LA CIUDAD DE TAPACHULA EN EL CONTEXTO DEL CORREDOR MigRATORIO CENTROAMÉRICA-MÉXICO-EsTAdos UNIDOS}

La ciudad de Tapachula es la cabecera municipal del municipio que lleva el mismo nombre, es el principal centro económico y administrativo de la región X Soconusco. ${ }^{2}$ Es uno de los principales pases fronterizo para población que proviene de Centroamérica y de otras regiones como África y el Caribe. Esta ciudad es además uno de los espacios urbanos más importante del corredor migratorio Centroamérica-México. Por su dinámica económica y administrativa es una ciudad conectada con el orden económico global. La ciudad funciona como el centro administrativo de la región agroindustrial cafetalera del Soconusco, desde donde se realizan transacciones económicas y comerciales que la conectan con las dinámicas de los mercados internacionales, pues es una de las principales regiones proveedoras de café de empresas transnacionales (Espinosa, 2018). Asimismo, es la ciudad mexicana que encabeza la lista en la Encuesta Nacional de Seguridad Pública de población (96 por ciento) que considera que vivir en su ciudad es insegura (INEGI, 2018).

Por su ubicación geoestratégica fronteriza, en la ciudad existen consulados de los países centroamericanos, así como oficinas de la Organización

1 Se integra por actividades relacionadas con el trabajo doméstico y de cuidados (INEGI, 2017).

2 La región X Soconusco comprende los municipios de Acacoyagua, Acapetahua, Cacahuatán, Escuintla, Frontera Hidalgo, Huhuetán, Huixtla, Mazatán, Metapa, Villa Comaltitlán, Suchiate, Tapachula, Tuxtla Chico, Tuzantán y Unión Juárez (CEIEG, 2012). 
Internacional para las Migraciones (OIM), ACNUR y diversas Organizaciones de la Sociedad Civil que defienden los derechos humanos de las personas migrantes, por lo que su visibilidad en la literatura especializada es recurrente.

Un importante número de investigaciones han puesto en evidencia la importancia de esta ciudad en la frontera sur de México, se ha documentado la heterogeneidad de las migraciones que arriban y transitan por Tapachula (Nájera, 2016); los efectos de la política migratoria restrictiva basada en el control (Anguiano, 2008); las crisis de derechos humanos y las violencias que experimentan quienes migran y llegan a la ciudad (Porraz, 2017; París, 2017); los nichos laborales en las tareas domésticas y de cuidados de las mujeres guatemaltecas (Blanco, 2014); igualmente, se han desarrollado algunas investigaciones que analizan la presencia de niñas, niños y adolescentes inmigrantes en la ciudad y sus vulnerabilidad frente al trabajo y la trata de personas (López, 2018; Ceriani y Gómez, 2014; Casillas, 2006).

La ciudad de Tapachula es el destino principal de la niñez migrante de origen guatemalteco, indígena en su mayoría. Si bien, mucha de la población de 0 a 17 años de dicho país llega a territorio chiapaneco bajo la modalidad de migración familiar para emplearse en las fincas agrícolas de la región del Soconusco, también un grupo importante de niños y niñas tienen por destino principal la ciudad de Tapachula donde se emplean en actividades propias del comercio y los servicios (López, 2018; Ayala y Cárcamo, 2012; Álvarez, 2010).

En el contexto de la crisis migratoria de los últimos años, algunas organizaciones de la sociedad civil han detectado la presencia de niñas y adolescentes inmigrantes mayoritariamente indígenas guatemaltecas que se incorporan a trabajos domésticos y de cuidados en hogares a terceros, en ciudades fronterizas como Tapachula, Chiapas y Tenosique, Tabasco, asimismo, ha detectado nuevas rutas migratorias de estas niñas y adolescentes, para viajar a ciudades turísticas del caribe mexicano y emplearse en trabajos domésticos y de cuidados (Lagunes y Barraza, 2016). Estas organizaciones han registrados las principales características de las niñas y las adolescentes inmigrantes que se incorporan al trabajo doméstico:

Originarias principalmente del departamento de San Marcos, en Guatemala son, en su mayoría, indígenas mayas mam, provenientes de familias campesinas. Desde muy pequeñas, y debido a las condiciones de violencia estructural que experimentan cotidianamente, estas mujeres cruzan la frontera hacia Tapachula, Chiapas para acompañar a sus padres en el trabajo estacional agrícola. Posteriormente, y con una edad aproximada de 14 años, comienzan a migrar de 
manera individual para así aportar tanto a necesidades familiares como buscar sueños personales (Lagunes y Barraza, 2016: 2).

Incluso durante el trabajo de campo hubo dos relatos de autoridades locales donde se afirmó que a partir de los años noventa, la arquitectura de las viviendas en la ciudad de Tapachula cambió para dar cabida al servicio doméstico, se empezaron construir casas con una habitación adicional destinada a una o más empleadas, como un señal de estatus y distinción entre las familias tapachultecas, pues les resultó barato contar con una empleada para las labores domésticas dado el aumento de la oferta de mujeres jóvenes inmigrantes de Guatemala.

Cabe señalar que la niñez y juventud indígena mam migrante proviene mayoritariamente de los departamentos guatemaltecos Huehuetenango, Quetzaltenango y San Marcos. Estos departamentos cuentan con una historia caracterizada por violencias estructurales traducidas en altos índices de pobreza, economía basada en la agricultura de subsistencia y un pasado reciente marcado por la guerra civil que generó desplazamientos internos e internacionales (Castillo y Toussaint, 2015).

Según un reciente informe de UNICEF (2016), en Guatemala los hogares con mayor incidencia de pobreza multidimensional son los hogares con población indígena (76.4 por ciento) sobre todo si se les compara con los hogares mestizos (54.4 por ciento). Las mayores deficiencias que enfrentan los hogares indígenas se presentan en acceso a servicios de salud, condiciones de las viviendas y acceso a la educación (UNICEF, 2016).

En tanto que "Ocho de cada diez niños y adolescentes indígenas viven en situación de pobreza, de los cuales 84.9 por ciento viven en pobreza y 45.5 por ciento en pobreza extrema" (UNICEF, 2016). Ello se refleja en diversas carencias que afectan sobre todo a quienes habitan en zonas rurales. Un ejemplo es la desnutrición crónica que perjudica en mayor medida a la niñez indígena (61.2 por ciento) en relación con la no indígena (34.5 por ciento); las dificultades se acentúan por condición de género, las niñas y las adolescentes pues la tasa de educación es menor en las mujeres pues de cada diez niñas, únicamente seis terminan la escolaridad primaria (UNICEF, 2016).

Este panorama de violencias estructurales ha sido enfrentado mediante la estrategia de movilidad hacia las principales ciudades fronterizas tales como Huixtla y Tapachula. La Encuesta sobre Migraciones en la Frontera Sur 2017 da cuenta que del total de personas entrevistadas provenientes de 
Guatemala 4.5 por ciento tenían entre 15 y 17 años de edad. ${ }^{3}$ De los cuales 83 por ciento eran hombres y 17 por ciento mujeres; 22 por ciento dijo que pertenecía a algún pueblo o comunidad indígena; 99 por ciento dijo que iba a buscar trabajo en México y 29 por ciento contestó que ya había trabajado en su lugar de origen. Destaca que 45.9 por ciento de los hombres viajan con el objetivo de buscar trabajo en el sector agropecuario y 28.6 por ciento en el sector de la construcción; en tanto las mujeres contestaron en su mayoría (70.5 por ciento) que pensaban buscar trabajo en el servicio doméstico y 20.1 por ciento en algún comercio (COLEF et al., 2017).

Esta realidad tiene lugar en el contexto de las recientes tendencias globales hacia el rechazo de las migraciones y la profundización de discursos xenofóbicos. En la ciudad de Tapachula es común escuchar expresiones tales como "tenemos plaga de migrantes, son una plaga", "estamos llenos de esa gente", "llegan y llegan los mareros y tatuados" entre otras, con cargas xenofóbicas y estereotipadas por nacionalidad, incluso trabajadores de la administración municipal y del Desarrollo Integral de la Familia (DIF) se expresan de los inmigrantes como un mal que aqueja a la ciudad (información obtenida en trabajo de campo). Al tiempo que los discursos anti migrantes proliferan en los medios de comunicación y se endurecen las políticas y medidas de control en la frontera (Rocha, 2019).

Ha sido ampliamente documentado que esta ciudad es un nodo importante para el crimen organizado, donde los inmigrantes pueden ser víctimas de delitos diversos, desde robos, lesiones, trata de personas y secuestros, además de abusos por parte de las fuerzas de orden público. Tal es el grado de la problemática que se ha calificado la región y la ciudad como geografías de la violencia y/o paisajes de terror (París, 2017 y Porraz, 2017).

En este contexto de hostilidad y violencias estructurales, niñas y adolescentes indígenas provenientes de Guatemala, se concentran por lo regular en el parque central "Miguel Hidalgo"4 que ha sido uno de los principales lugares de reunión, trabajo y esparcimiento entre la niñez y la juventud inmigrante, al tiempo que se ha consolidado como el principal espacio de encuentro entre empleadoras/es y niñas, adolescentes y mujeres que buscan emplearse en el trabajo doméstico (Álvarez, 2010).

\footnotetext{
${ }^{3}$ Incluye únicamente a las personas entrevistadas, esta cifra no contempla a los acompañantes de menos de 15 años de edad.

4 "El Miguel Hidalgo es uno de los espacios urbanos más concurridos y más dinámicos comercial, financiera, política y culturalmente en Tapachula. En uno de sus extremos se ubica la sede del Ayuntamiento Municipal, y en el otro una zona de restaurantes. Alrededor del parque se hallan algunas edificaciones históricas y centros culturales, como la iglesia de San Agustín o el antiguo Palacio Municipal, donde actualmente funciona la Casa de Cultura y el Museo Arqueológico del Soconusco (Alvarez, 2010: 133).
} 
Trabajo infantil doméstico y violencias en la ciudad de Tapachula, Chiapas 2017-2018 / S. MIRANDA JUÁREZ

\section{NiÑAS Y ADOLESCENTES EN EL TRABAJO DEL HOGAR Y VIOLENCIAS RACIALES}

Conocer la magnitud y las condiciones de la participación de las niñas y adolescentes inmigrantes en el trabajo doméstico es un reto que se enfrenta desde el sector académico y las instituciones del Estado encargadas de velar por el bienestar de las infancias y las adolescencias. Por su condición migratoria de alta movilidad es complejo identificarles y darles seguimiento en sus trayectos, en tanto que por su condición laboral caracterizada por la informalidad y a puertas cerradas, la identificación y cuantificación es aún más difícil.

Por ello en esta investigación, se pusieron en marcha algunas herramientas metodológicas propias del enfoque cualitativo para obtener datos de primera mano. Se realizaron tres estancias en la ciudad de Tapachula entre 2017 y 2018. Para la identificación y contacto con las niñas y las adolescentes trabajadoras del hogar se visitaron tres mercados: El Mercado Sebastián Escobar, el Mercado San Juan y el Tianguis Tapachula para conversar con los administradores y empleadoras acompañadas por empleadas. Se realizaron también rondas y caminatas por las siguientes colonias: Los Naranjos, Centro, Del Maestro, Lomas del Soconusco, Fraccionamiento Jaime Sabines Gutiérrez, Fraccionamiento Antorcha III y Los Tulipanes donde se identificaron letreros que solicitaban empleada a fin de entrevistar tanto a empleadoras como empleadas. Se aplicaron entrevistas semi estructuradas a autoridades del Consulado de Guatemala, del Centro de Atención a Niñas, Niños y Jóvenes Migrantes del sistema DIF Municipal, trabajadoras sociales del Hospital General, directora de la Asociación Civil por la Superación de la Mujer, responsables del área de mujeres y niñas inmigrantes del Centro de Derechos Humanos Fray Matías de Córdoba, así como observación participante en la casa de mujeres T’ja Xujm, en la Plaza Cristal y en el parque central Miguel Hidalgo.

Se entrevistó a un total de diez niñas y adolescentes; siete procedentes de Guatemala, dos originarias de El Salvador y una procedente de Honduras en un rango de 11 a 16 años de edad. Todas ellas trabajadoras del hogar, ocho de ellas en modalidad de trabajadora de planta y dos en modalidad de trabajadora de entrada por salida. Cabe señalar que la niña de menor edad (11 años) procedía de El Salvador. No fue posible entrevistar niños y adolescentes varones a pesar de que durante el trabajo de campo salió a la luz la presencia de estos en el trabajo del hogar. Las siete niñas y adolescentes de Guatemala eran hablantes de lengua indígena mam. Cuatro de 
ellas terminaron únicamente la escolaridad primaria y tres estaban en alguna etapa de la escolaridad básica (escolaridad secundaria), ellas afirmaron que estaba trabajando para poder ahorrar y así hacer frente a los costos de la educación diversificada (bachillerato).

Las dos chicas de El Salvador se empleaban como trabajadoras del hogar al tiempo que trabajaban en el negocio de su empleador - un desayunador y un puesto de comidas en el Mercado San Juan - al igual que la chica que provenía de Honduras quien trabajaba en una tienda de abarrotes en el mismo mercado. Las diez entrevistadas dijeron que viajaron a Tapachula sin documento migratorio y lo hicieron por diferentes rutas que permiten las entradas y salidas informales. Tres de ellas (originarias de Guatemala) dijeron que su primera experiencia migratoria y laboral había sido años atrás en las fincas del café en modalidad de migración laboral familiar.

En su mayoría, las niñas y adolescentes inmigrantes que buscan emplearse en el trabajo del hogar en la ciudad de Tapachula, dijeron que buscan empleo por medio de hermanas mayores, primas o amigas con experiencias previas que las invitan y les facilitan el acceso al trabajo, les brindan consejos y se convierten en sus redes sociales de apoyo. Llegar a buscar empleo en los mercados de la ciudad es una de las principales estrategias para colocarse primero en algunos negocios como cafeterías y comedores y posteriormente entrar al servicio doméstico. El trabajo de planta les representa ventajas para poder ahorrar el pago del alquiler y los alimentos (Tabla 1).

Por su parte, las empleadoras entrevistadas fueron 14. No todas tiene relación con las niñas y adolescentes entrevistadas. Siete fueron entrevistadas en los mercados visitados, tres se entrevistaron a partir de las caminatas en las colonias y la identificación de letreros en las puertas o ventanas de las viviendas. Dos fueron entrevistadas en sus negocios (una papelería y una heladería) y dos en la Plaza Comercial Cristal. Sus ocupaciones son variadas: empresarias, comerciantes del mercado, profesoras, médicas, empleadas administrativas en las oficinas del Ayuntamiento, amas de casa. La principal razón expresada para emplear a chicas en el trabajo del hogar fue la necesidad de ayuda con las labores que implican tener hijo/as (Tabla 2).

Se identificaron diversas violencias que enfrentan las niñas y las adolescentes indígenas de Guatemala que se emplean a puertas cerradas en hogares de terceros. A continuación, se analizan los relatos derivados de las entrevistas. 
Trabajo infantil doméstico y violencias en la ciudad de Tapachula, Chiapas 2017-2018 / S. MIRANDA JUÁREZ

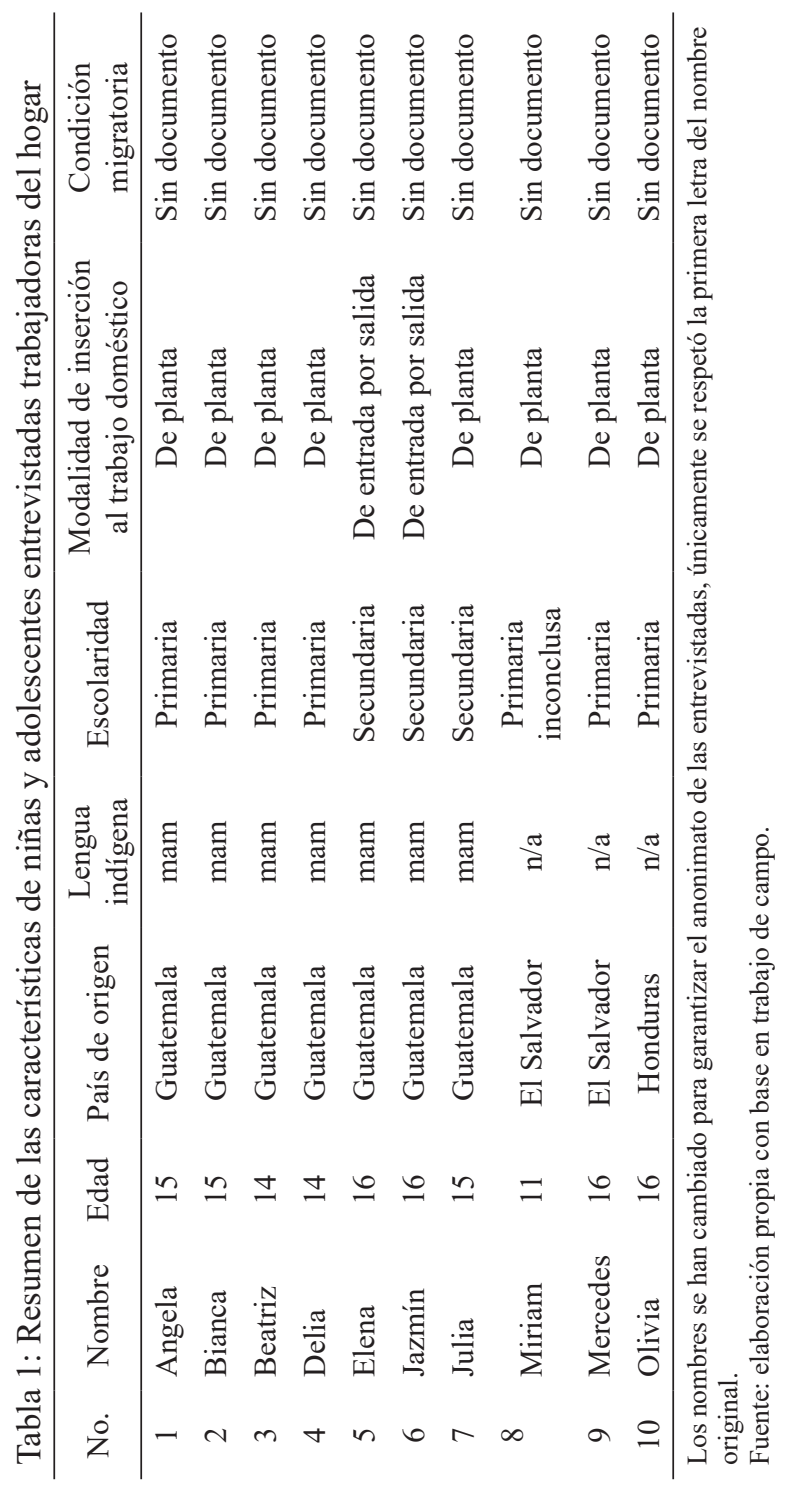




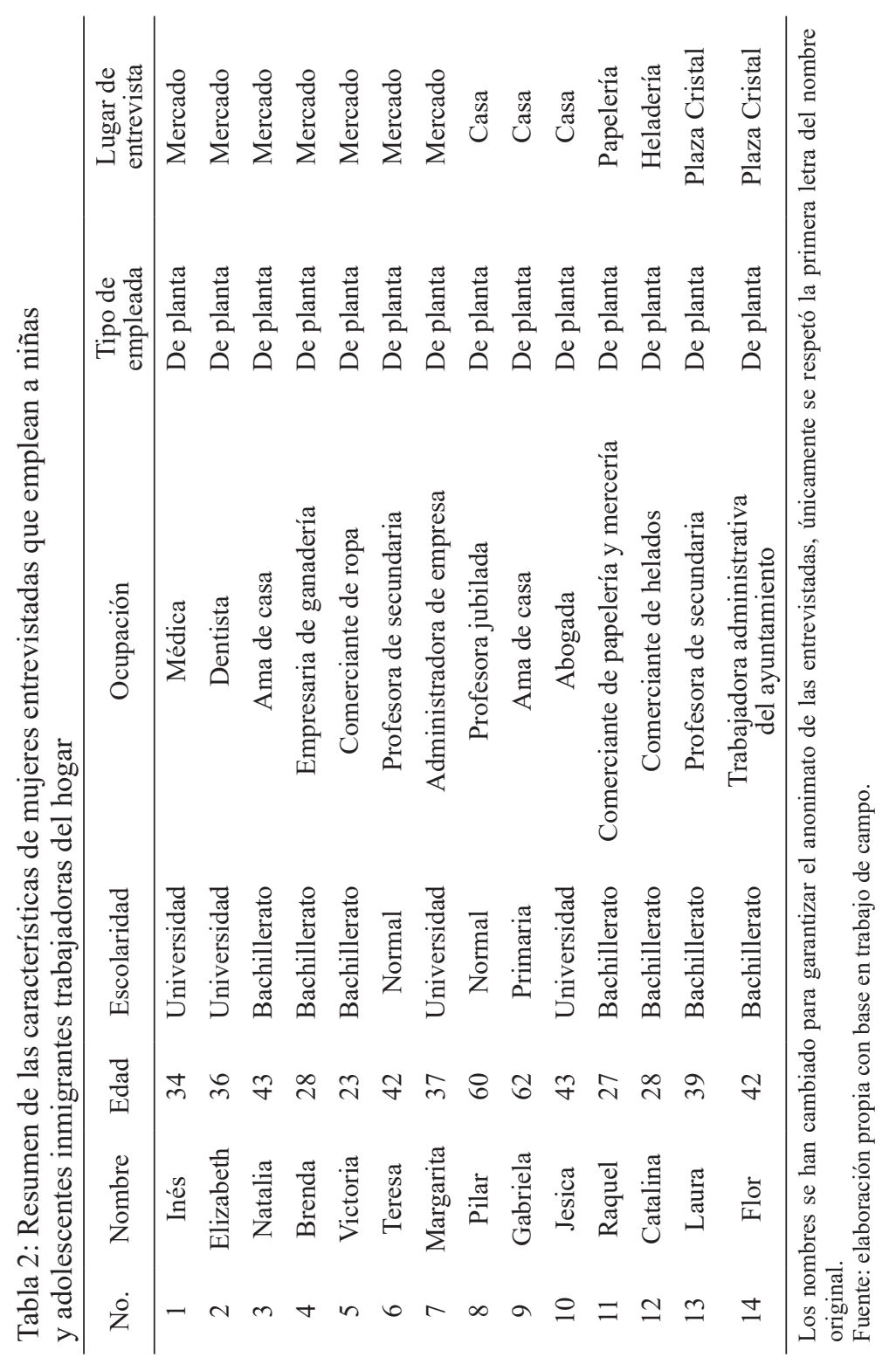




\section{LAS VIOLENCIAS EN EL CONTEXTO DEL TRABAJO DOMÉSTICO}

Las niñas y las adolescentes migrantes que trabajan en Tapachula en hogares de terceros enfrentan una serie de violencias vinculadas a las circunstancias estructurales de sus comunidades de donde proceden, signadas por pobreza y desigualdad. Cuentan con escaso acceso a la educación básica y en la mayoría de los casos mencionaron la violencia intrafamiliar, comunitaria y de género como un importante factor para tomar la decisión de migrar hacia Tapachula y buscar la autonomía económica.

En tanto que al llegar a México e insertarse en el trabajo doméstico experimentan otras violencias legitimadas a partir de las categorías que portan tales como el género, la edad, la etnia y la condición migratoria.

En relación con la edad, las chicas mencionaron diversas violencias. Las empleadoras les mencionan que son menores de edad y a partir de ello justifican los amenazas y malos tratos:

“...no me gusta mucho el trato a veces, te gritan y te acusan, te ponen más ropas de lavar, te insultan, que por gordita, que porque hago las cosas lenta, y me tengo que enseñar, porque dijo la señora que es muy peligroso que soy una menor, no tengo los 18, no me conviene que se enteren en el gobierno o en un abogado por eso dice que mejor no le responda feo, yo mejor no le respondo a nada que me diga, puede acusar, no me conviene, no me igualo con ella..." (Elena, 16 años, trabajadora de entrada por salida, entrevistada en el Fraccionamiento Jaime Sabines Gutiérrez).

El relato anterior expresa el adultoscentrismo en las prácticas de la empleadora, quien detenta el poder frente a una persona de menor edad -que supuestamente desconoce los procesos y debe ser instruida-, con ello de legitima la exigencia de obediencia y la aceptación de malos tratos en función de la edad, este argumento fue también expuesto por algunas empleadoras:

"son muchachitas y se les tiene que decir con mano dura que una es la que se arriesga... no tienen la edad, no muy saben lo que quieren, requieren madurez para hacer las cosas, que se les guíe, se les enseñe bien, yo por eso les enseño todo, me fijo, pero es difícil, a veces mi marido le tiene que hablar fuerte, es regaño sí, son palabras fuertes ¿verdad? pero es necesario, sino no pues le toman a una la medida desde bien chicas" (Gabriela, 62 años, Empleadora colonia Centro).

Por la vía de la condición migratoria se encontraron también algunas violencias, que se interseccionan con la condición étnica de las niñas y las 
adolescentes. Como afirma Pavez (2018) algunos grupos de inmigrantes son objeto de múltiples violencias en función de sus diferencias fenotípicas, vestimenta y lengua, derivado de un proceso de racialización en el que los inmigrantes se perciben como inferiores frente a la población local.

Las autoridades consulares de Guatemala dieron cuenta del vínculo entre condición migratoria y étnica, y su efecto sobre las violencias que experimentan las niñas y las adolescentes. Ser migrantes sin documentos legales que les pone en mayor vulnerabilidad frente a las violencias incluso físicas. Como se expresa en el relato siguiente, existe personas que se adjudican el poder de "civilizar" a las indias (Cumes, 2014), mediante violencia física:

"hay de todo... aquí hay hasta políticos, jueces, eh... funcionarios públicos y hogares que tal vez no tienen los recursos para vivir muy cómodos pero como se les hace fácil contratarlas y decir me robaste entonces las tienen de manera gratuita, pero recuerdo mucho las palabras de un juez, una vez fuimos por el tema de una niña golpeada, y nos dijo que deberíamos de dar las gracias a él, que porque gracias a ellos, ellas tienen trabajo aunque sean inditas y de Guatemala... entonces... una me quede así como que si eso dice un juez, que puedo esperar de la sociedad ¿no?’ (Autoridad consular de Guatemala).

La racialización a la que se enfrentan ciertos grupos de inmigrantes, resulta en el ejercicio de diversas violencias y utiliza argumentos del orden de lo biológico y natural para ejercer agresiones y ofensas (Pavez, 2018). Así como las nociones de asco e higiene (Cumes, 2014) generan estereotipos que a su vez exponen a las niñas inmigrantes a la violencia verbal por parte de las empleadoras y sus familias. Los siguientes relatos dan cuenta de tal situación:

"lo que no me gusta es que la señora me dice del olor, dice que no me baño, y que no lavo mi corte. ${ }^{5}$ Me burla, me regaña, me habla mal cuando uso mi corte, de mi pelo que es sucio y que no quiere que caiga en la comida, pero lo amarro, y me dio jabón, eso no me gusta, no, el corte no es sucio, pero ella me dice" (Beatriz, 14 años, trabajadora de planta, entrevistada en colonia el Naranjo).

"La señora no me dice nada, solo me dice qué hacer, pero lo que es su hija, sí, ella me dice varios insultos, me dice que tengo mugre, que no hablo bien, que las de Guatemala no hablamos bien y no se bañan, se ríe, es muy lista no crea que lo hace en frente a la señora no, me lo dice cuando pasa y estoy barriendo o lavando, yo digo que es de persona grosera" (15 años, trabajadora de planta, colonia Los Tulipanes).

\footnotetext{
5 Traje típico.
} 
Por su parte, la racialización en función de la exotización de los cuerpos salió a la luz en varias ocasiones, las empleadoras hablaron reiteradamente sobre la preferencia de nacionalidad guatemalteca frente a otras como la salvadoreña u hondureña, su justificación está fundada en ideas tales como las características fenotípicas y de personalidad sumida y humilde, características que son consideradas de valía frente al trabajo doméstico, pues como señala la literatura especializada, el trabajo doméstico ha estado históricamente asociado al servilismo:

“...de hecho sí, yo ocupo, miro chicas que todavía vienen con sus trajes típicos, al momento de emplearse o llegar al parque, la mayoría de ellas ya se lo quita y vemos que ya vienen "transformadas" de alguna manera, porque, porque no... igual y no les da confianza a las persona que ya las van a emplear, a mi sí, porque yo les veo su traje típico y digo "esta si me va a funcionar" y ya me la llevo a mi casa, porque del Salvador, o sea o de más de Centroamérica ya no me la llevaría, es considerada un poco más agresiva, ... mmm no sé, porque vienen más de Centroamérica, no fácil le doy entrada a mi casa" (Natalia, 43 años, empleadora entrevistada en Mercado San Juan).

Como contraparte, los relatos de las niñas y las adolescentes muestran los insultos y las humillaciones como la forma en que se materializa la racialización en el contexto laboral, ante la pregunta sobre alguna situación que les haga sentir sufrimiento o dolor en su trabajo, contestaron lo siguiente:

"de primero me hacía sentir triste lo de mi corte, lo que decía que mejor me vistiera normal, luego me dijo que mejor mi corte porque con pantalón iba a parecer como de Honduras que van para los bares, es que como que no le gusta una cosa o la otra cosa no le gusta, y me burla de mi corte y me burla de mi pantalón y así es cuando se enoja, ...o si a veces no hago bien las cosas dice que mejor me vaya para los bares con los hombres porque no lo hago bien, que soy como de Honduras, sí, eso no me gusta mucho" (Angela, 15 año, trabajadora de planta, entrevistada en Mercado Sebastián Escobar).

"los insultos de venir de Guate no me gustan, tampoco que me diga "la María", no es así mi nombre, y se hace de la burla, yo les digo mi nombre y ríen" (Jazmín, 16 años, trabajadora de entrada por salida, entrevistada en Parque Miguel Hidalgo).

El siguiente relato es quizá uno de los que ilustra de mejor manera la suma de violencias normalizadas a partir de un proceso de racialización que se combina con la edad y el origen migratorio en el contexto del traba- 
jo doméstico. Ante la pregunta de los criterios para contratar a una empleada doméstica, la empleadora estableció el siguiente argumento:

"Mire ¿cómo le diré? una es por la fisionomía ¿En qué sentido? Que no sean muy atractivas ¿verdad?, ... entonces no tengo temor de que me quieran quitar a mi marido (risas), sí... esa es una... vienen de pueblos que lo aguantan todo... ¿sí? ... entonces... si yo le pongo a cargar a una niña, la pongo a cargar cien sillas que las pase para acá, sé que va a aguantar porque viene del monte, es india, viene de la montaña, si es capaz de cargar un quintal de leña pues puede cargar esto, entonces es esta cuestión, al ser más niñas puedo pedirle hacer más cosas, lamentablemente es una cuestión que me importa mucho porque tengo mis niños, que me cuide a los niños en la noche ¿sí? entonces las niñas son... más dóciles en ese sentido, y sí, probablemente voy aceptar a una salvadoreña, pero a quien no voy a aceptar como trabajadora del hogar es a una niña hondureña..." (Gabriela, 62 años, empleadora entrevistada en su casa).

Sumado a lo anterior, el interjuego de estas características indentitarias, al imbricarse con el género pueden resultar en la combinación idónea para el ejercicio de violencias, sobre todo de orden sexual. Si bien ninguna de las niñas y adolescentes entrevistadas hablaron directamente de agresiones sexuales; autoridades consulares y miembros de organizaciones de defensa de derechos humanos dieron cuenta del recurrente abandono de adolescentes indígenas procedentes de Guatemala en los principales hospitales públicos de la ciudad al momento de dar a luz, como resultado de abuso o supuesto enamoramiento de hijos o sobrinos de las empleadoras:

"muchas veces son engañadas por personas que buscan trabajo, muchas veces son engañadas por las señoras, les quitan sus hijos a las niñas, luego hasta las sacan de la casa, creo que hace un mes tuvimos el caso de una niña que la golpeaban a la guatemalteca, la dejaron en el hospital con su bebé y ya pusimos la denuncia en contra de la supuesta suegra que era la patrona, porque eso era, la patrona, y la niña que decía que yo quiero ver al muchacho, hijo de la señora misma jimagínese! y para saber, ya no se responsabilizaron, ahí la engañaron, ya quedó la niña con semejante problema, esa muchachita tenía 15 años, imagínese a qué edad la embarazó este muchacho" (Autoridad consular de Guatemala).

\section{CONCLusiones}

A partir de la información anterior, se lograron estructurar algunas reflexiones finales, a fin de seguir complejizando y discutiendo la práctica 
del trabajo infantil doméstico. Sobre todo, en un contexto local y regional atravesado por la problemática de la violencia en frontera.

Si bien, la práctica del trabajo infantil es considerada una afrenta a los derechos de las niñas y los niños, en este contexto se identificó que las niñas y adolescentes indígenas que salen de sus comunidades de origen hacia la ciudad de Tapachula, lo hacen por decisión personal ante una escasa calidad de vida y en la mayoría de los casos por la vivencia de violencias domésticas en sus hogares y comunidades de origen.

Al llegar a la ciudad de Tapachula, se insertan en el nicho de mercado del trabajo doméstico donde por sus características identitarias (etnia, edad, género, condición migratoria) son fácil blanco de otras violencias en el ámbito ocupacional. Por lo que podría hablarse de un continuum de violencias que experimenta a lo largo de su vida, en distintas esferas como la familiar y laboral.

Aunque este fenómeno no es nuevo, las cadenas globales del cuidado y del trabajo doméstico, encuentran nuevas formas para seguir reproduciendo un orden social, donde las niñas y las adolescentes encarnan la subordinación frente a ciertos grupos que renuevan discursos de miedo, inseguridad y xenofobia para obtener trabajo a bajo costo. Ello es posible, mediante el ejercicio de diferentes violencias que como se vio en los relatos, encuentran su justificación en la interacción entre edad, raza y condición migratoria.

Estudiar a las niñas y adolescente indígenas e inmigrantes que se emplean en las distintas modalidades del trabajo doméstico en un contexto regional como el de la ciudad de Tapachula representó un reto teórico y metodológico para generar conocimiento sobre las experiencias laborales de este grupo de población y las violencias que enfrentan. Asimismo, se logró visibilizar la complejidad en que se articulan y operan los engranajes del sexismo, el racismo, la xenofobia y el adultoscentrismo para la preservación del orden social, donde las niñas y las adolescentes asumen el costo de trabajar y vivir en territorios y espacios domésticos diferentes a su hogar de origen al margen de la protección social con alto riesgo frente a los abusos y las violencias.

Ante ello, cabe preguntarse ¿Qué tipo sociedad permite que un hombre abuse a puerta cerrada de una adolescente indígena e inmigrante que trabaja como empleada del hogar?, ¿Qué factores permiten que esa adolescente de 15 años sea abandonada con su hijo en un hospital público al momento de dar a luz? ¿Qué tipo de acciones emprenden los gobiernos 
nacionales y locales ante la problemática del trabajo infantil doméstico a puertas cerradas?

La complejidad de este fenómeno exige respuestas y políticas integrales que muestren un interés genuino en la protección y garantía de los derechos de las niñas y adolescentes que se encuentran en los márgenes geográficos y simbólicos. De lo contario, difícilmente se podrán modificar las estructuras sociales que mantienen a ciertas infancias en condiciones de desventaja y vulnerabilidad social.

\section{REFERENCIAS BIBLIOGRáFICAS}

Alvarenga, T., Benítez, M. y Walder, J., 2005, Antiguas costumbres, prácticas nuevas: intervenciones frente al criadazgo en el siglo XXI, Global... Infancia, Fondo para la Igualdad de Género (FIG)-Paraguay: Agencia Canadiense de Desarrollo Internacional (ACDI).

Álvarez, Soledad, 2010, “A la sombra del Miguel Hidalgo: análisis etnográfico del parque central de Tapachula", en LiminaR, San Cristóbal de las Casas, vol. 8, n . 2, p. $129-152$.

Anderson, B., 2000, Doing the Dirty Work?: The Global Politics of Domestic Labour, London y New York: Zed Books.

Anguiano, M. E., 2008, “Chiapas: Territorio de Inmigración, emigración y tránsito migratorio", en Papeles de Población No. 56. México: Universidad Autónoma del Estado de México.

Arriagada, I. y Moreno, M., 2011, "La constitución de cadenas globales de cuidado y las condiciones laborales de las trabajadoras peruanas en Chile", en Stefoni C., Mujeres inmigrantes en Chile: ¿Fuerza de trabajo o mujeres con derechos?, Santiago de Chile: Universidad Alberto Hurtado.

Association al Kara, 2009, Rapport Annuel 2008 Pour la protection del'enfant en situation précaire. Marrakech.

Ayala, M. y Cárcamo, N., 2012, "Los niños y niñas guatemaltecas migrantes en la frontera sur de México: Acompañantes o trabajadores", en Ra Ximhai, vol. 8, núm. 1, enero-abril.

Barot, R. y Bird J., 2001, "Racialization: The genealogy and critique of a concept", in Ethnic and Racial Studies 24 (4): 601-618.

Benítez, M., 2013, "La OIT denuncia las condiciones deplorables del trabajo doméstico", en Revista Digital ABC, en https://www.abc.es/sociedad/20130109/abci-trabajo-domestico-201301091125.html (consultado el 3 de octubre de 2019).

Blanco, B., 2014, "Negociación y resistencia: relaciones diádicas en el empleo doméstico de mujeres guatemaltecas en Tapachula, Chiapas", en Durin, S. et al. (Coords.), Trabajadoras en la sombra: dimensiones del servicio doméstico latinoamericano, México: Ediciones de la Casa Chata. 
Borjas, G., 1999, "The economic analysis of immigration", Chapter 28 in Handbook of Labor Economics, 1999, vol. 3, Part A, pp 1697-1760.

Bustamante, J., 2008, Promotion and Protection of all Human Rights, Civil, Political, Economic Social and Cultural Rights, Including the Rigth to Development. Report of the special Rapporteur on the Human Rights of Migrants. Addendum (Mission to Mexico) Ginebra: Human Rights Counsil, Undécima sesión. Agenda item 3, 9-15 de marzo.

Carcedo, A., 2004, “Desde niñas, mujeres invisibles: el trabajo infantil doméstico en hogares de terceros", en Sagot, Marcela (comp.), Una mirada de género al trabajo infantil doméstico, Costa Rica: Programa Internacional para la Erradicación del Trabajo Infantil, Oficina Internacional del Trabajo.

Casillas R., 2006, La trata de mujeres, adolescentes, Niñas y niños en México. Un estudio exploratorio en Tapachula, Chiapas, México: Comisión Interamericana de Mujeres de la Organización de Estados Americanos/OIM/INMUJERES/INM.

Castillo, M. y Toussaint, M., 2015, “La frontera sur de México: orígenes y desarrollo de la migración centroamericana", en Cuadernos Inter.c.a.mbio sobre Centroamérica y el Caribe, vol. 12, No. 2 julio-diciembre.

CEIEG, 2012, Regiones Socioeconómicas de Chipas, Comité Estatal de Información Estadística y Geográfica de Chiapas disponible en http://www.ceieg.chiapas. gob.mx/productos/files/CIGECH/CIGECH_REGIONES.pdf

Ceriani Cernadas, P. y Gómez Salas, A., 2014, "Niñez y adolescencia en el contexto de la migración: principios, avances y desafíos en la protección de sus derechos en América Latina y el Caribe", en REMHU - Rev. Interdiscipl. Mobil. Hum., año 22, nº. 42, p. 9-28. Brasilia.

COLEF, STPS, CONAPO, SRE, CONAPRED, 2017, Encuesta sobre Migración en la Frontera Sur de México, El Colegio de la Frontera Norte, Secretaría del Trabajo y Previsión Social, Consejo Nacional de Población, Unidad de Política Migratoria, Secretaría de Relaciones Exteriores, Consejo Nacional para Prevenir la Discriminación, disponible en http://www.colef.mx/emif.

Cortez, D., Cáceres, C., y Venegas, R., 2005, Diagnóstico general de los trabajadores temporales en la frontera sur de México, México: CEM-INM.

Cumes, A., 2014, "La casa como espacio de civilización", en Durin, S. et al. (Coords.), Trabajadoras en la sombra: dimensiones del servicio doméstico latinoamericano, México: Ediciones de la Casa Chata.

Díaz M., 2008, "El mercado de trabajo de los cuidados y la creación las cadenas: ¿Cómo concilian las cuidadoras?”, en Cuadernos de Relaciones Laborales, vol. 26, $\mathrm{n}^{\circ}$. 2, Universidad Complutense de Madrid.

Durin, S., 2017, Yo trabajo en casa. Trabajo del hogar, género y etnicidad en Monterrey, México: Ediciones de la Casa Chata.

Espinosa M., 2018, Los empresarios cafetaleros del Soconusco, Chiapas, frente a la globalización, Tesis que para obtener el grado de Doctor en Estudios Regionales, Tuxtla Gutiérrez, Chiapas: Universidad Autónoma de Chiapas. 
Glaser, B. and Strauss, A., 1967, The discovery of grounded theory. Chicago: Aldine Press.

Glockner, V., 2008, De la montaña a la frontera: identidad, representaciones sociales y migración de los niños mixtecos de Guerrero. El Colegio de Michoacán.

Hatton, T. y Williamson, J., 1994, "International Migration: 1850-1939: An Economic Survey", in Migration and the International Labor Market 1850-1939, London: Routledge: 3-32.

Hondagneu, P., 2011, Doméstica. Trabajadoras inmigrantes a cargo de la limpieza y el cuidado a la sombra de la abundancia. México: Instituto Nacional de Migración.

Human Rights Watch, 2005, "Inside the Home, Outside the Law Abuse of Child Domestic", in Workers in Morocco 17, $\mathrm{n}^{\circ} .12$ (E).

INEGI, 2017, Módulo de Trabajo Infantil, Encuesta Nacional de Ocupación y Empleo, México. México: INEGI.

INEGI, 2018, Encuesta Nacional de Seguridad Pública. Principales Resultados. México: INEGI.

International Monetary Fund, 1994, Human Capital Flight: Impact of Migration on Income and Growth, IMF, Working Paper, No 94/ 155, Washington, D.C.

KidsRights Foundation, 2014, Behind Closed Doors. Child Domestic Labour, with a focus on the Kamlari system in Nepal, Holland: KidsRights Foundation and Leiden University.

Lagunes, A. y Barraza, R., 2016, Cuidar de las que cuidan: mujeres migrantes y trabajo del hogar en la frontera México-Guatemala, disponible en http://ichan. ciesas.edu.mx/puntos-de-encuentro/cuidar-de-las-que-cuidan-mujeres-migrantes-y-trabajo-del-hogar-en-la-frontera-mexico-guatemala/ (Consultado el 23 de agosto de 2019).

Llorent, V., 2013, "Las "Petites Bonnes” marroquíes: causas y consecuencias socioeducativas", en, Educatio Siglo XXI, vol. 31 n ${ }^{\circ}$. 1, pp. 335-356.

López, Y., 2018, "El trabajo infantil: de la discusión teórica a la realidad etnográfica. Motivaciones de la infancia guatemalteca para trabajar en Tapachula, Chiapas", en EntreDiversidades, (11), 137-165. Disponible en https://doi.org/10.31644/ ED.11.2018.a05

Martínez, V., 2014, "Niñez, migración y derecho: aportes para un abordaje antropológico", en Revista Sociedad and Equidad, núm. 6, enero, pp. 237-257.

Miranda, S., 2016, "Imaginarios a futuro de niños jornaleros de Villa Guerrero", en Olvera, J. y Baca, N. (Coords.) Continuidades y cambios en las migraciones de México a Estados Unidos. Tendencias en la circulación, experiencias y resignificaciones de la migración y el retorno en el Estado de México, Universidad Autónoma del Estado de México y Universidad de Texas.

Muñoz, C. y Ochoa, J., 2010, "Estructuras Familiares en Hogares con Criaditas/ os: un Estudio Cualitativo", en Eureka: 7(1): 66-83, Asunción, Paraguay. 
Nájera J., 2016, "El complejo estudio de la actual migración en tránsito por México: Actores, temáticas y circunstancias", en Migraciones internacionales, vol. 8 $\mathrm{n}^{\circ} .3$ Tijuana: El Colegio de la Frontera Norte.

OIM, 2018, Informe sobre las Migraciones en el Mundo 2018, Organización Internacional para las Migraciones.

OIT-IPEC, 2002, Perú Invisible y sin derechos: Aproximación al perfil del trabajo infantil doméstico, Lima, Perú: OIT-IPEC.

París, M. D., 2017, Violencias y migraciones centroamericanas en México, Baja California México: El Colegio de la Frontera Norte, A.C.

Parreñas, R., 2005, Long distance intimacy: Class, gender and intergenerational relations between mothers and children in Filipino transnational families. Global Networks, 4, 317-336.

Pavez, I., 2018, "Violencias contra la infancia migrante en Santiago de Chile: Resistencias, agencia y actores", en Migraciones Internacionales, vol. 9, núm. 4, julio-diciembre.

Porraz, I., 2017, "Entender las violencias: los jóvenes migrantes centroamericanos en sus lugares de origen y su tránsito por el sur de México", en Nueva antropología, vol. 30, nº. 87, pp.107-130.

Ramellini, T., 2004, "El trabajo doméstico de niñas y adolescentes como violación de Derechos Humanos y factor de riesgo para la violencia", en Sagot, M. (Comp.), Una mirada de género al trabajo infantil doméstico, San José, Costa Rica: Programa Internacional para la Erradicación del Trabajo Infantil, Oficina Internacional del Trabajo.

Ramírez, N., 2009, Del Matatero tero lá al Matarile rile ro: Infancia migrante en Tapachula, México: Ririki Intervención Social, S.C.

Rocha, J. L., 2019, Políticas migratorias estadounidenses y resistencias de los centroamericanos indocumentados en la era de Trump, Guatemala: Universidad Rafael Landívar, Editorial Cara Parens.

Rodríguez, C., 2007, La organización del cuidado de niños y niñas en Argentina y Uruguay, CEPAL, Serie Mujer y desarrollo N 90, Santiago de Chile.

Sagot, M., 2004, Una mirada de género al trabajo infantil doméstico, San José, Costa Rica: Programa Internacional para la Erradicación del Trabajo Infantil, Oficina Internacional del Trabajo.

Solimano, A., 2001, International Migration and the Global Economic Order: An Overview, Working Paper, No 2720, Washington, D.C.: Banco Mundial.

Tijoux M. E. y Palominos S., 2015, "Aproximaciones teóricas para el estudio de procesos de racialización y sexualización en los fenómenos migratorios de Chile", en Polis, Revista Latinoamericana, vol. 14, nº . 42, p. 247-275

Tijoux, M.A., 2013, "Niños(as) marcados por la inmigración peruana: estigma, sufrimientos, resistencias", en Convergencia. Revista de Ciencias Sociales, vol. 20, nº. 61, enero-abril, 2013, pp. 83-104. 
UNICEF, 2016, Pobreza Multidimensional Infantil y Adolescente en Guatemala: Privaciones a superar, Guatemala: UNICEF.

\section{RESUMEN CURRICULAR DE LA AUTORA}

Sarai Miranda Juárez

Investigadora Cátedra CONACYT-ECOSUR en el proyecto 435 Violencias de género y desigualdad en el sureste de México. Licenciada en Economía por la Universidad Autónoma del Estado de México; Maestra en Ciencias Sociales con especialidad en Desarrollo Municipal por El Colegio Mexiquense y Doctora en Estudios de Población por El Colegio de México. En el año 2017 ganó la Beca a las Mujeres en Ciencias Sociales de la Academia Mexicana de Ciencias. SNI Nivel I. Realizó estancias de investigación en la Universidad de Buenos Aires y en la Universidad Complutense de Madrid, fue Investigadora invitada el Instituto de Investigaciones Gino Germani de la Universidad de Buenos Aires.

Dirección electrónica: saraimirandaj@gmail.com

Registro ORCID: http://orcid.org/0000-0003-1387-0497 